\title{
ON LAVES' GRAPH OF GIRTH TEN
}

\author{
H. S. M. COXETER
}

1. Introduction. This note shows how a certain infinite graph of degree three, discovered by Laves in connection with crystal structure, can be inscribed (in sixteen ways, all alike) in an infinite regular skew polyhedron which has square faces, six at each vertex. One-eighth of the vertices of the polyhedron are vertices of the graph, and the three edges of the graph that meet at such a vertex are diagonals of alternate squares. Thus either diagonal of any face of the polyhedron can serve as an edge, and the whole graph can then be completed in a unique manner.

The same graph is also derived, by the method of Frucht's paper (4), from the abstract group

$$
S_{1}^{2} S_{2} S_{1}^{2}=S_{2}, \quad S_{2}{ }^{2} S_{1} S_{2}^{2}=S_{1}
$$

2. A regular skew polyhedron. In $1926, \mathrm{~J}$. F. Petrie discovered an infinite regular skew polyhedron which can be derived from the simple honeycomb of cubes $\{4,3,4\}$ by taking all the vertices, all the edges, and half the squares (1, pp. $33-35 ; 2$, p. $242 ; 6$, p. 55 , Fig. 4). This new regular polyhedron was named $\{4,6 \mid 4\}$ because it has square faces, six at each vertex, and square holes corresponding to the missing faces of the cubic honeycomb.

The vertex figure of a cube $\{4,3\}$ is an equilateral triangle $\{3\}(3$, p. 16$)$; the vertex figure of the cubic honeycomb $\{4,3,4\}$ is an octahedron $\{3,4\}$ (3, p. 68); and the vertex figure of the skew polyhedron $\{4,6 \mid 4\}$ is a skew hexagon which is a Petrie polygon of the octahedron (3, pp. 24-25). Since a given octahedron has four Petrie polygons, the faces of the skew polyhedron can be selected from those of a given cubic honeycomb in four ways. Taking the vertices to be all the points whose Cartesian coordinates are integers, one way is select the squares

$$
\begin{aligned}
& (0,0,0)(0,1,0)(0,1,-1)(0,0,-1) \\
& (1,0,0)(1,1,0)(1,1,-1)(1,0,-1)
\end{aligned}
$$

and all others that can be derived from these two by permuting the three coordinates and adding fixed even numbers to them.

3. The infinite group $\{R, S\}$. In saying that the skew polyhedron is "regular," we mean that it possesses two special symmetry operations: $R$, cyclically permuting the vertices of one face (say the former of those mentioned above), and $S$, cyclically permuting the faces at one vertex (say the

Received June 8, 1954. 
origin). These operations generate an infinite group having the elegant abstract definition

$$
R^{4}=S^{6}=(R S)^{2}=\left(R S^{-1}\right)^{4}=1
$$

$(1$, pp. 35,48$)$. Since they are rotatory reflections $(1$, p. 37$)$, their squares are pure rotations: $R^{2}$, of period 2 about the "axis" of the square

$$
(0,0,0)(0,1,0)(0,1,-1)(0,0,-1) \text {, }
$$

and $S^{2}$, of period 3 about the line $x=y=z$. In fact, $R^{2}$ is the transformation

$$
x^{\prime}=x, \quad y^{\prime}=1-y, \quad z^{\prime}=-1-z,
$$

while $S^{2}$ is the cyclic permutation

$$
x^{\prime}=z, \quad y^{\prime}=x, \quad z^{\prime}=y .
$$

4. The subgroups $\left\{T_{1}, T_{2}, T_{3}\right\}$ and $\left\{S_{1}, S_{2}, S_{3}\right\}$. It is convenient to let $T_{1}, T_{2}, T_{3}$ denote $R^{2}$ and its transforms by $S^{2}$ and $S^{-2}$, namely

$$
\begin{aligned}
& T_{1}: \quad x^{\prime}=x, \quad y^{\prime}=1-y, \quad z^{\prime}=-1-z, \\
& T_{2}: \quad x^{\prime}=-1-x, y^{\prime}=y, \quad z^{\prime}=1-z, \\
& T_{3}: \quad x^{\prime}=1-x, \quad y^{\prime}=-1-y, z^{\prime}=z .
\end{aligned}
$$

We use $S_{1}, S_{2}, S_{3}$ to denote the products $T_{2} T_{3}, T_{3} T_{1}, T_{1} T_{2}$, namely

$$
\begin{aligned}
& S_{1}: \quad x^{\prime}=2+x, \quad y^{\prime}=-1-y, z^{\prime}=1-z, \\
& S_{2}: \quad x^{\prime}=1-x, \quad y^{\prime}=2+y, \quad z^{\prime}=-1-z \text {, } \\
& S_{3}: \quad x^{\prime}=-1-x, y^{\prime}=1-y, \quad z^{\prime}=2+z .
\end{aligned}
$$

Thus $S_{1}{ }^{2}, S_{2}{ }^{2}, S_{3}{ }^{2}$ increase the respective coordinates by 4 , and $S_{1} T_{2} S_{3}$ increases each of them by 2 .

The half-turns $T_{i}$ can be expressed in an obvious way as products of pairs of reflections $A_{i}$ and $B_{i}$, namely

$$
T_{1}=A_{3} B_{2}, \quad T_{2}=A_{1} B_{3}, \quad T_{3}=A_{2} B_{1},
$$

where

$$
\begin{aligned}
& A_{1} \text { is } \quad x^{\prime}=-1-x, y^{\prime}=y, \quad z^{\prime}=z \text {, } \\
& B_{1} \text { is } \quad x^{\prime}=1-x, \quad y^{\prime}=y, \quad z^{\prime}=z \text {, } \\
& A_{2} \text { is } x^{\prime}=x, \quad y^{\prime}=-1-y, z^{\prime}=z \text {, }
\end{aligned}
$$

and so on. Thus the $A$ 's and $B$ 's are reflections in the opposite faces

$$
x=\mp \frac{1}{2}, \quad y=\mp \frac{1}{2}, \quad z=\mp \frac{1}{2}
$$

of the cube $\left( \pm \frac{1}{2}, \pm \frac{1}{2}, \pm \frac{1}{2}\right)$. They generate the direct product of three infinite groups

$$
A_{i}{ }^{2}=B_{i}{ }^{2}=1 \quad(i=1,2,3),
$$

since both $A_{i}$ and $B_{i}$ commute with both $A_{j}$ and $B_{j}$ whenever $i \neq j$. The 
products $A_{i} B_{i}$ increase the respective coordinates by 2 . We easily verify that

and

$$
\left(A_{i} B_{i}\right)^{2}=S_{i}^{2}
$$

$$
A_{1} B_{1} A_{2} B_{2} A_{3} B_{3}=A_{1} A_{2} A_{3} B_{1} B_{2} B_{3}=T_{2} T_{3} T_{2} T_{1} T_{2}=S_{1} T_{2} S_{3} .
$$

5. An infinite graph. Since the three $T$ 's are half-turns about three edges of the above-mentioned cube, which are the axes of three faces of $\{4,6 \mid 4\}$ (see Fig. 1), they transform the origin into the three points

$$
(0,1,-1), \quad(-1,0,1), \quad(1,-1,0)
$$

which are the opposite vertices of these squares. Thus the group $\left\{T_{1}, T_{2}, T_{3}\right\}$ transforms the origin into the points

$$
\begin{array}{llll}
(0,0,0), & (1,2,3), & (2,3,1), & (3,1,2) \\
(2,2,2), & (3,0,1), & (0,1,3), & (1,3,0)
\end{array}
$$

$(\bmod 4)$,

which are derived from $(0,0,0)$ and $(1,2,3)$ by cyclically permuting the coordinates, adding 2 to all of them, and adding arbitrary multiples of 4 to any of them.

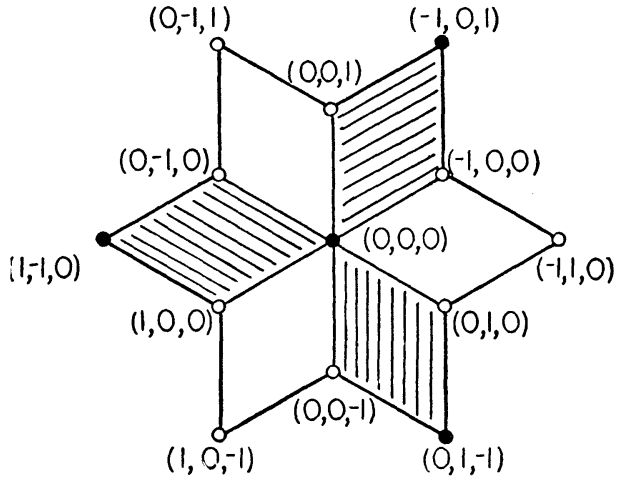

Figure 1

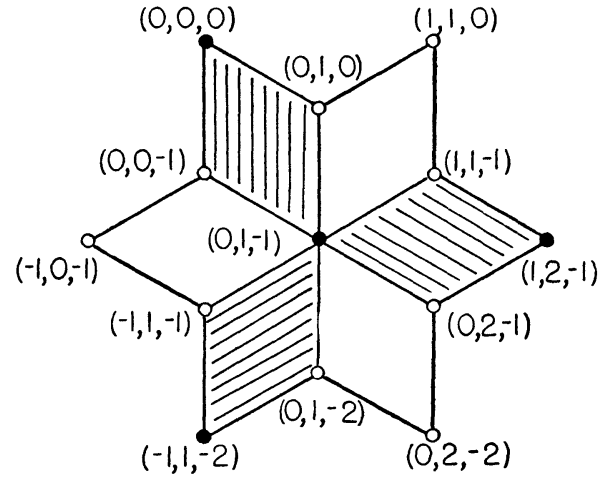

Figure 2

In this manner we pick out one-eighth of the vertices of $\{4,6 \mid 4\}$, occurring at the ends of diagonals of one-eighth of the squares (shaded in Figs. 1 and 2 ). These diagonals are the edges of a graph of degree three which is the Cayley colour group for $\left\{T_{1}, T_{2}, T_{3}\right\}$. It is remarkable that the three edges at a vertex are coplanar and meet at angles of $120^{\circ}$, like those of the plane tessellation of hexagons, $\{6,3\}$.

The smallest circuit in this graph is a skew decagon such as

$$
\begin{gathered}
(0,0,0) \quad(0,1,-1) \quad(1,2,-1)(1,3,0)(2,3,1) \quad(2,2,2) \quad(1,2,3) \quad(0,1,3) \\
(-1,1,2)(-1,0,1)
\end{gathered}
$$


In other words, the graph is of girth ten (9, p. 459). This property corresponds to the fact that, apart from

$$
T_{1}^{2}=T_{2}{ }^{2}=T_{3}{ }^{2}=1,
$$

the simplest relations satisfied by the $T$ 's are

$$
T_{1} T_{2} T_{1} T_{3} T_{1}=T_{2} T_{3} T_{2} T_{1} T_{2}=T_{3} T_{1} T_{3} T_{2} T_{3}
$$

(i.e., $S_{3} T_{1} S_{2}=S_{1} T_{2} S_{3}=S_{2} T_{3} S_{1}$, or $S_{i}{ }^{2} T_{i}=T_{i} S_{i}{ }^{2}$ ), each involving ten $T$ 's.

In terms of the $S^{\prime}$ 's alone, which evidently satisfy

these relations become

$$
S_{1} S_{2} S_{3}=1,
$$

$$
S_{1}{ }^{2} S_{2} S_{1}{ }^{2}=S_{2}, \quad S_{2}{ }^{2} S_{3} S_{2}{ }^{2}=S_{3}, \quad S_{3}{ }^{2} S_{1} S_{3}{ }^{2}=S_{1}
$$

(any two of which imply the remaining one). The first implies

$$
S_{1}{ }^{2} S_{2}{ }^{2}=S_{1}{ }^{2} S_{2} \cdot S_{1}{ }^{2} S_{2} S_{1}{ }^{2}=S_{1}{ }^{2} S_{2} S_{1}{ }^{2} \cdot S_{2} S_{1}{ }^{2}=S_{2}{ }^{2} S_{1}{ }^{2} ;
$$

thus the squares of the $S$ 's all commute with one another. By repeated application of

$$
\begin{array}{lll}
S_{1} S_{2}{ }^{2}=S_{2}{ }^{-2} S_{1}, & S_{2} S_{3}{ }^{2}=S_{3}{ }^{-2} S_{2}, & S_{3} S_{1}{ }^{2}=S_{1}{ }^{-2} S_{3}, \\
S_{1} S_{3}{ }^{2}=S_{3}{ }^{-2} S_{1}, & S_{2} S_{1}{ }^{2}=S_{1}{ }^{-2} S_{2}, & S_{3} S_{2}{ }^{2}=S_{2}{ }^{-2} S_{3},
\end{array}
$$

we can transform any "word" into the standard form

$$
S_{1}{ }^{x} S_{2}{ }^{y} S_{3}{ }^{z}
$$

where at most one of $x, y, z$ is odd; e.g.,

$$
S_{1} S_{3}=S_{1}{ }^{2} S_{1}{ }^{-1} S_{3}{ }^{-1} S_{3}{ }^{2}=S_{1}{ }^{2} S_{2} S_{3}{ }^{2} .
$$

Thus the Abelian subgroup $\subseteq=\left\{S_{1}{ }^{2}, S_{2}{ }^{2}, S_{3}{ }^{2}\right\}$, having the four cosets

$$
\text { ธ, } \subseteq S_{1}, \subseteq S_{2}, \subseteq S_{3},
$$

is of index 4 in $\left\{S_{1}, S_{2}, S_{3}\right\}$.

6. A finite graph. The abstract group $\left\{S_{1}, S_{2}, S_{3}\right\}$ has a finite factor group

$$
\begin{gathered}
S_{1}{ }^{2 l}=S_{2}{ }^{2 m}=S_{3}{ }^{2 n}=S_{1} S_{2} S_{3}=1, \\
S_{1}{ }^{2} S_{2} S_{1}{ }^{2}=S_{2}, \quad S_{2}{ }^{2} S_{3} S_{2}{ }^{2}=S_{3}, \quad S_{3}{ }^{2} S_{1} S_{3}{ }^{2}=S_{1}
\end{gathered}
$$

or

$$
S_{1}{ }^{2 l}=S_{2}{ }^{2 m}=\left(S_{1} S_{2}\right)^{2 n}=1, \quad S_{1}{ }^{2} S_{2} S_{1}{ }^{2}=S_{2}, \quad S_{2}{ }^{2} S_{1} S_{2}{ }^{2}=S_{1},
$$

whose order is $4 l m n$ (since it has a subgroup $\mathfrak{E}_{l} \times \mathfrak{C}_{m} \times \mathfrak{E}_{n}$ of index 4 ). The case $l=m=n$ has been described by Frucht $(4,5.51)$.

Geometrically, since the squared $S$ 's in the infinite group are translations, the effect of giving them definite periods $l, m, n$ is to identify points whose three coordinates differ by multiples of $4 l, 4 m, 4 n$, respectively. In other words, the infinite space is reduced to a three-dimensional torus. Since the order of 
the finite group is $4 l m n$, the corresponding graph has $8 l m n$ vertices and $12 l m n$ edges.

The girth is still 10 , provided $l, m, n$ are all greater than 2 . But if $l=2$, the relation $S_{1}{ }^{4}=1$ or $\left(T_{2} T_{3}\right)^{4}=1$, involving 8 letters, yields an 8 -circuit. In the special case $l=m=n=2$, every edge belongs to just two 8-circuits (e.g., the " $T_{2}$ " edge belongs to $\left(T_{1} T_{2}\right)^{4}=1$ and $\left.\left(T_{2} T_{3}\right)^{4}=1\right)$; therefore the graph $(4,5.23)$ can be embedded in a surface to form a regular map of 24 octagons.

7. A thin packing of spheres. Returning to the infinite graph (with $l, m, n$ unrestricted), we note that something essentially equivalent to it was discovered in 1932 by Laves (7, p. 10). When the graph is derived from the $\{4,6 \mid 4\}$ whose vertices have integral coordinates, its edges are of length $\sqrt{ } 2$. Hence spheres of diameter $\sqrt{ } 2$, drawn around all the vertices, will touch one another at the mid-points of the edges. This arrangement of spheres is the figure described by Laves. His Fig. 7 shows, in a very striking manner, some of the rings of ten spheres corresponding to 10-circuits in the graph.

Since there are eight spheres for each cube of edge 4 , the packing density is

$$
8 \cdot \frac{4}{3} \pi\left(\sqrt{ } \frac{1}{2}\right)^{3} / 4^{3}=\frac{\pi}{12 \sqrt{ } 2}=0.18512 \ldots
$$

namely one-quarter that of the cubic or hexagonal close-packing. Nevertheless, this is not the thinnest possible packing of equal spheres. By the simple but ingenious device of replacing each sphere by a cluster of three smaller ones (5, pp. 448-450; 8, p. 484), Heesch and Laves derived a still thinner packing, with density only

$$
\pi \sqrt{ } 2\left(\sqrt{ } 3-\frac{3}{2}\right)^{3}=0.055515 \ldots
$$

8. A correction. I take this opportunity to correct an unfortunate error in my paper on Regular skew polyhedra (1, pp. 54, 55, 61). The regular map shown in Fig. Xv is not $\{4,7 \mid 3\}$ but $\{4,6 \mid 3\}$. Moreover, Table II should be supplemented by three further entries:

\begin{tabular}{|c|r|r|r|r|r|r|}
\hline Polyhedron & \multicolumn{1}{|c|}{$f$} & \multicolumn{1}{|c|}{$e$} & \multicolumn{1}{|c|}{$v$} & \multicolumn{1}{|c|}{$p$} & \multicolumn{1}{|c|}{$g$} \\
\cline { 2 - 6 }$\{4,6 \mid, 2\}$ & 12 & 24 & 8 & 3 & $S_{4} \times S_{2}$ & 48 \\
$\{5,6 \mid, 2\}$ & 24 & 60 & 20 & 9 & $A_{5} \times S_{2}$ & 120 \\
$\{3,11 \mid, 4\}$ & 2024 & 3036 & 552 & 231 & $L F(2,23)$ & 6072 \\
\hline
\end{tabular}

Note added in proof. When applied to the four-dimensional polyhedron $\{4,6 \mid 3\}$ (1, pp. 45,55$)$, the procedure of $\$ \S 1$ and 5 (using diagonals of alternate squares at a vertex) yields a graph of girth five having 20 vertices and 30 
edges (one diagonal of each square); this is the same as the graph formed by the vertices and edges of the regular dodecahedron $\{5,3\}$. When applied to $\{4,6 \mid, 2\}$, it yields the complete 4-point (i.e., the vertices and edges of the tetrahedron $\{3,3\})$. When applied to $\{4,6 \mid, 3\}(1, \mathrm{p} .60)$, it yields a graph of girth six having 24 vertices and 36 edges (diagonals of 36 of the 84 squares). This graph, denoted by $\{12\}+\{12 / 5\}(4,4.1)$, consists of the vertices and edges of the map $\{6,3\}_{2,2}$ on a torus, and is the Cayley colour group for the octahedral group $S_{4}$ generated by the three transpositions $T_{i}=(i 4)$ $(i=1,2,3)$.

\section{REFERENCES}

1. H. S. M. Coxeter, Regular skew polyhedra in three and four dimensions, and their topological analogues, Proc. London Math. Soc. (2), 43 (1937), 33-62.

2. - The regular sponges, or skew polyhedra, Scripta Math., 6 (1939), 240-244.

3. - - Regular polytopes (London, 1948; New York, 1949).

4. R. Frucht, Remarks on finite groups defined by generating relations, Can. J. Math., 7 (1955), 8-17.

5. H. Heesch and F. Laves, Über dünne Kugelpackungen, Z. Kristallogr., 85 (1933), 443-453.

6. H. Jensen, De seks uendelige regulaere polyedre, Mat. Tidsskr. (A), 1950, 53-60.

7. F. Laves, Zur Klassifikation der Silikate, Z. Kristallogr., 82 (1932), 1-14.

8. S. Melmore, The densest and the least dense packings of equal spheres, Mineralogical Magazine, 28 (1949), 479-485.

9. W. T. Tutte, A family of cubical graphs, Proc. Cambridge Philos. Soc., 43 (1947), 459-474.

University of Toronto 